\title{
ON PRIME IDEALS WITH GENERIC ZERO $x_{i}=t^{{ }^{n}}{ }_{i}$
}

\section{H. BRESINSKY}

ABSTRACT. Let $n_{i}, 1 \leq i \leq r, r \geq 3$, be natural numbers such that $\left(n_{1}, \cdots, n_{r}\right)=1$ and $n_{i} \stackrel{i}{=} \Sigma_{j=1}^{r} z_{j} n_{j}, z_{j}$ nonnegative integers, implies $z_{j}=0, j \neq i$, and $z_{i}=1$. It is shown that for prime ideals with generic zero $x_{i}=t^{n}$ and $r \geq 4$, arbitrary large finite minimal sets of generators exist.

Let $Z-Z^{-}$denote the nonnegative integers, let $n_{i}$ be a natural number, $1 \leq i \leq r, r \geq 3$, and let $\left\langle n_{1}, \cdots, n_{r}\right\rangle$ be the additive semigroup $\{z \mid z=$ $\left.\Sigma_{i=1}^{r} z_{i} n_{i}, z_{i} \in Z-Z^{-}\right\}$. Assume further $\left(n_{1}, \cdots, n_{r}\right)=1$ and $n_{i} \notin\left\langle n_{1}, \cdots\right.$, $\left.\hat{n}_{i}, \cdots, n_{r}\right)$. Let $K$ be an arbitrary field and $P\left(n_{1}, \cdots, n_{r}\right)$ the prime ideal $\left\{f\left(x_{1}, \cdots, x_{r}\right) \mid f\left(x_{1}, \cdots, x_{r}\right) \in K\left[x_{1}, \cdots, x_{r}\right], f\left(t^{n_{1}}, \cdots, t^{n_{r}}\right)=0, t\right.$ transcendental over $K\}$. Let $\left|P\left(n_{1}, \cdots, n_{r}\right)\right|<\infty$ be the smallest number of generators of $P\left(n_{1}, \cdots, n_{r}\right)$, and let $P(r)=1$.u.b. $\left\{z|z=| P\left(n_{1}, \cdots, n_{r}\right) \mid\right\}$. In [2] it is shown that for $r=3, P(3)=3$ and $\left|P\left(n_{1}, n_{2}, n_{3}\right)\right|=2$ iff $\left\langle n_{1}, n_{2}, n_{3}\right\rangle$ is symmetric. The purpose of this note is to prove the following statement.

Theorem. $r \geq 4 \Rightarrow P(r)=\infty$.

The proof is by induction on $r$, hence initially $r=4$. Let $q_{1}=q_{2}+1$, $q_{2}$ even, $q_{2} \geq 4, d_{1}=q_{2}-1$. Set $n_{1}=q_{1} q_{2}, n_{2}=q_{1} d_{1}, n_{3}=q_{1} q_{2}+d_{1}, n_{4}=$ $q_{2} d_{1} \cdot\left(n_{1}, n_{2}, n_{3}, n_{4}\right)=1, n_{h} \notin\left\langle\hat{n}_{h}, n_{i}, n_{j}, n_{k}\right\rangle$, $A_{1}=\left\{f_{\mu} \mid f_{\mu}=x_{1}^{\mu-1} x_{3}^{q_{2}-\mu}-x_{2}^{q_{2}-\mu} x_{4}^{\mu+1}, 1 \leq \mu \leq q_{2}\right\} \subseteq P\left(n_{1}, n_{2}, n_{3}, n_{4}\right)=P$, $\left\{g_{1}=x_{1}^{d}-x_{2}^{q_{2}}, g_{2}=x_{3} x_{4}-x_{2} x_{1}\right\} \subseteq P$ follows readily.

Lemma 1. $\alpha_{h} n_{h} \in\left\langle\hat{n}_{h}, n_{i}, n_{j}, n_{k}\right\rangle, \alpha_{h}$ minimal $\Rightarrow \alpha_{1}=d_{1}, \alpha_{2}=q_{2}, \alpha_{3}=$ $d_{1}$, and $\alpha_{4}=q_{1}$.

Proof. $\nu_{1} q_{1} q_{2}=\mu_{2} q_{1} d_{1}+\mu_{3}\left(q_{1} q_{2}+d_{1}\right)+\mu_{4} q_{2} d_{1} \Rightarrow \nu_{1}>\mu_{3}$, and

Presented to the Society, October 15, 1973; received by the editors November 13, 1973.

AMS (MOS) subject classifications (1970). Primary 13C05; Secondary 14 H45. 
$d_{1} \mid \nu_{1}-\mu_{3} \Rightarrow \nu_{1} \geq d_{1}$. The other proofs are similar.

Let

$$
\begin{gathered}
A_{2}=\left\{f \mid f=x_{1}^{\nu_{1}} x_{4}^{\nu_{4}}-x_{2}^{\mu_{2}} x_{3}^{\mu_{3}}, \nu_{1}, \mu_{3}<d_{1}, f \in P\right\}, \\
A=A_{1} \cup A_{2} \cup\left\{g_{1}, g_{2}\right\}, A^{\prime}=A \cup\left\{f \mid f=x_{1}^{\nu_{1}} x_{4}^{\nu_{4}}-x_{2}^{\mu_{2}} x_{3}^{\mu_{3}}, f \in P\right\},
\end{gathered}
$$

and let $(A)$ and $\left(A^{\prime}\right)$ be the ideals generated by the respective sets.

Lemma 2. $\left(A^{\prime}\right)=P \Rightarrow(A)=P$.

Proof. Replace $x_{1}^{\nu}{ }_{1} x_{4}^{\nu}-x_{2}{ }_{2}{ }^{\mu} x_{3}{ }^{\mu} \in A^{\prime}, \nu_{1}, \mu_{3} \geq d_{1}$, by

$$
\begin{aligned}
& x_{1}^{\nu_{1}} x_{4}^{\nu_{4}}-x_{2}^{\mu_{2}} x_{3}^{\mu_{3}}-x_{1}^{\nu_{1}-d_{1}} x_{4}^{\nu}{ }_{4} f_{q_{2}}=x_{1}^{\nu_{1}-d_{1}} x_{4}^{\nu_{4}+q_{1}}-x_{2}^{\mu_{2}} x_{3}^{\mu_{3}} \text {. }
\end{aligned}
$$

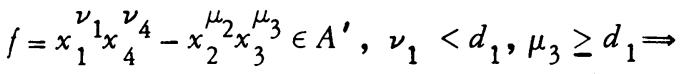

$$
\begin{aligned}
& x_{1}^{\nu_{1}} x_{4}^{\nu}{ }_{4}-x_{2}^{\mu}{ }_{2} x_{3}^{\mu}+x_{2}^{\mu} x_{3}{ }^{\mu}{ }^{-d}{ }_{1} f_{1}=x_{1}{ }_{1} x_{4}{ }_{4}{ }_{4}-x_{2}{ }_{2}+d_{1} x_{x_{3}} \mu_{3}-d_{1} x_{4}^{2} \text {. }
\end{aligned}
$$

$\nu_{1}<d_{1} \Rightarrow \nu_{4}>2 \Rightarrow f^{\prime}=x_{1}^{\nu_{1}} x_{4}^{\nu_{4}-2}-x_{2}^{\mu_{2}+d_{1}} x_{3} \mu_{3}-d_{1} \in A^{\prime}$. Replace $f$ by $f^{\prime}$.

Lemma 3. $\left(A^{\prime}\right)=P$.

Proof. By [2, Proposition 1.4], $P\left(n_{1}, \cdots, n_{r}\right)$ is generated by

$$
F(\nu, \mu)=x_{1}^{\nu_{1}} \ldots x_{r}^{\nu_{r}}-x_{1}^{\mu_{1}} \ldots x_{r}^{\mu_{r}}, \quad \partial(F(\nu, \mu))=\sum_{i=1}^{r} \nu_{i} n_{i}=\sum_{i=1}^{r} \mu_{i} n_{i},
$$

$\nu_{i}$ or $\mu_{i}=0,1 \leq i \leq r$. The existence of $f \in\left(A^{\prime}\right) \quad F(\nu, \mu)-f=$ $\Pi_{i=1}^{4} x_{i}{ }^{\delta_{i}} g, g=0$ or $g=F\left(\nu^{\prime}, \mu^{\prime}\right), \partial\left(F\left(\nu^{\prime}, \mu^{\prime}\right)\right)<\partial(F(\nu, \mu))$ will prove the lemma. If in $F(\nu, \mu), \nu_{3}=\mu_{3}=0$, then $F(\nu, \mu) \in P \cap K\left[x_{1}, x_{2}, x_{4}\right] .\left\langle n_{1}, n_{2}, n_{4}\right\rangle$ symmetric and [2] $\Rightarrow P \cap K\left[x_{1}, x_{2}, x_{4}\right]=\left(g_{1}, f_{q_{2}}\right) \subseteq\left(A^{\prime}\right)$. Hence assume $\nu_{3} \neq 0$.

(1) $\nu_{1} \doteq \nu_{2}=\nu_{4}=0 \Rightarrow \nu_{3} \geq d_{1} \cdot \mu_{2}=\mu_{4}=0 \Rightarrow \mu_{1} \geq d_{1}$ and $f=$ $-x_{1}^{\mu_{1}-d_{1}} g_{1}+x_{3}^{\nu_{3}-d_{1}} f_{1} \cdot \mu_{2}$ or $\mu_{4} \neq 0 \Rightarrow f=x_{3}^{\nu_{3}-d_{1}} f_{1}$.

(2) Exactly one $\nu_{j} \neq 0, j \neq 3 . \quad \nu_{2} \neq 0 \Rightarrow F(\nu, \mu) \in\left(A^{\prime}\right) . \quad \nu_{4} \neq 0 \Rightarrow f=$ $x_{3}^{\nu_{3}-1} x_{4}^{\nu_{4}-1} g_{2}$. Let $\nu_{1} \neq 0 . \nu_{3} \geq d_{1} \Rightarrow f=x_{1}^{\nu_{1}} x_{3}^{\nu_{3}-d}{ }^{-d} f_{1}$. 
PRIME IDEALS WITH GENERIC ZERO $x_{i}=t^{n}$

$$
\begin{aligned}
& \nu_{1} \geq d_{1} \Rightarrow f=x_{1}^{\nu_{1}-d_{1}} x_{3}^{\nu_{3}} g_{1} \quad \text { or } f=x_{1}^{\nu_{1}-d_{1}} x_{3}^{\nu_{3}} f_{q_{2}} \text {. } \\
& \nu_{1}, \nu_{3}<d_{1} \Rightarrow F(\nu, \mu)=x_{1}^{d_{1}-\sigma} x_{3}^{\nu_{3}}-x_{2}^{\mu_{2}} x_{4}^{\mu_{4}} \text {, }
\end{aligned}
$$

$\sigma$ and $\nu_{3}<d_{1} \Rightarrow\left(d_{1}-\sigma\right) q_{1} q_{2}+\nu_{3}\left(q_{1} q_{2}+d_{1}\right)=\mu_{2} q_{1} d_{1}+\mu_{4} q_{2} d_{1}$

$$
\Rightarrow d_{1} \mid d_{1}-\sigma+\nu_{3} \Rightarrow \sigma=\nu_{3} \Rightarrow f \in A_{1} \text {. }
$$

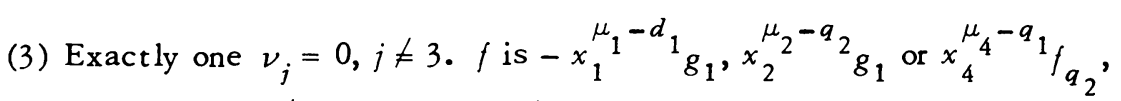
according to which $\mu_{j} \neq 0,1 \leq j \leq 4, j \neq 3$. Thus $(A)=P$.

Let $P^{e}$ be the extension of $P$ in $K\left\{x_{1}, x_{2}, x_{3}, x_{4}\right\}$, the formal ring of power series.

Lemma 4. $\bar{f}_{\mu} \in P^{e} / P^{e}\left(x_{1}, x_{2}, x_{3}, x_{4}\right), 1 \leq \mu \leq q_{2}$, are linearly independent over $K$.

Proof. Following a procedure similar to $[1$, p. 47], let $\phi$ be the substitution homomorphism $x_{1} \rightarrow T, x_{2} \rightarrow S^{q_{1}}, x_{3} \rightarrow T S, x_{4} \rightarrow S^{q_{2}}$. Then $\phi\left(g_{1}\right)=$ $T^{d}{ }^{1}-S^{q_{1}{ }^{q} 2}=w, \phi\left(f_{\mu}\right)=S^{q_{2}-\mu} w, 1 \leq \mu \leq q_{2}, \phi\left(g_{2}\right)=0$.

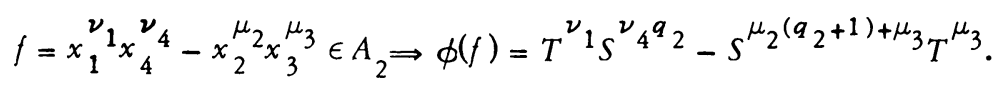

Since

$$
\begin{aligned}
\nu_{1} q_{1} q_{2}+\nu_{4} q_{2} d_{1} & =\mu_{2} q_{1} d_{1}+\mu_{3}\left(q_{1} q_{2}+d_{1}\right) \\
& \Leftrightarrow\left(\nu_{1}-\mu_{3}\right) q_{1} q_{2}=\left(\mu_{2} q_{1}+\mu_{3}-\nu_{4} q_{2}\right) d_{1} \Leftrightarrow \nu_{1}=\mu_{3}
\end{aligned}
$$

and $\nu_{4} q_{2}=\mu_{2} q_{1}+\mu_{3}, \phi(f)=0$. Applying $\phi$ to $\sum_{\mu=1}^{q_{2}} c_{\mu} f_{\mu} \in P^{e}\left(x_{1}, x_{2}, x_{3}, x_{4}\right)$, $c_{\mu} \in K$, gives $\sum_{\mu=1}^{q_{2}} c_{\mu} s^{q_{2}-\mu} \in \phi\left(x_{1}, x_{2}, x_{3}, x_{4}\right)$. Since any nonzero polynomial $f \in K[S] \cap \phi\left(x_{1}, x_{2}, x_{3}, x_{4}\right)$ has degree $\geq q_{2}, c_{\mu}=0,1 \leq \mu \leq q_{2}$.

Corollary 1. $P=\left(\pi_{1}, \cdots, \pi_{n}\right) \Rightarrow n \geq q_{2}$.

Corollary 2. $P(4)=\infty$.

Finally assume $P(r)=\infty, r \geq 4$. Let $\left|P\left(n_{1}, \cdots, n_{r}\right)\right|=m$.

Since $P\left(n_{1}, \cdots, n_{r}\right)$ is homogeneous in the $\left\langle n_{1}, \cdots, n_{r}\right\rangle$-graded ring $K\left[x_{1}, \cdots, x_{r}\right]$, and since $K$ is a field, by [3, Proposition 2 and Theorem 2], any two reduced sets of homogeneous generators are equal in number and minimal. Thus $\exists F\left(\nu_{i}, \mu_{i}\right), 1 \leq i \leq m$, generating $P\left(n_{1}, \cdots, n_{r}\right)$. Set $n_{i}^{\prime}=$ 
$2 n_{i}, 1 \leq i \leq r$, and let $n_{r+1}^{\prime}$ be a prime $>2 \partial\left(F\left(\nu_{i}, \mu_{i}\right)\right), 1 \leq i \leq m$. Again, since $P\left(n_{1}^{\prime}, \cdots, n_{r+1}^{\prime}\right)$ is homogeneous in the $\left\langle n_{1}^{\prime}, \cdots, n_{r+1}^{\prime}\right\rangle$-graded ring $K\left[x_{1}, \cdots, x_{r+1}\right]$, and since $n_{r+1}^{\prime}>\partial\left(F\left(\nu_{i}, \mu_{i}\right)\right), F\left(\nu_{i}, \mu_{i}\right) \in P\left(n_{1}^{\prime}, \cdots, n_{r+1}^{\prime}\right)$ $\cap K\left[x_{1}, \cdots, x_{r}\right], 1 \leq i \leq m,\left|P\left(n_{1}^{\prime}, \cdots, n_{r+1}^{\prime}\right)\right| \geq m$ and $P(r+1)=\infty$ follows by [3].

\section{REFERENCES}

1. H. Grauert and R. Remmert, Analytische Stellenalgebren, Springer-Verlag, Berlin and New York, 1971.

2. J. Herzog, Generators and relations of abelian semigroups and semigroup rings, Manuscripta Math. 3 (1970), 175-193. MR 42 \#657.

3. M. Nicolini, Sulle basi minimali di un ideale, Matematiche (Catania) 25 (1970), 174-181 (1971). MR $46 \# 3496$.

DE PARTMENT OF MATHEMATICS AND ASTRONOMY, UNIVERSITY OF MAINE, ORONO, MAINE 04473 